\title{
Instabilities and resistance fluctuations in thin accelerated superconducting rings
}

\author{
Mikko Karttunen, ${ }^{1,2}$ K. R. Elder, ${ }^{3}$ Martin B. Tarlie, ${ }^{4}$ and Martin Grant ${ }^{1}$ \\ ${ }^{1}$ Department of Physics and Centre for the Physics of Materials, McGill University, 3600 rue University, Montréal, \\ Québec, Canada H3A 2T8 \\ ${ }^{2}$ Biophysics and Statistical Mechanics Group, Laboratory of Computational Engineering, \\ Helsinki University of Technology, P.O. Box 9203, FIN-02015 HUT, Finland \\ ${ }^{3}$ Department of Physics, Oakland University, Rochester, Michigan, 48309-4487 \\ ${ }^{4}$ James Franck Institute, University of Chicago, 5640 South Ellis Avenue, Chicago, Illinois 60637
}

(Received 21 November 2001; published 23 August 2002)

\begin{abstract}
The nonequilibrium properties of a driven quasi-one-dimensional superconducting ring subjected to a constant electromotive force (emf) is studied. The emf accelerates the superconducting electrons until the critical current is reached and a dissipative phase slip occurs that lowers the current. The phase-slip phenomena is examined as a function of the strength of the emf, thermal noise, and normal state resistivity. Numerical and analytic methods are used to make detailed predictions for the magnitude of phase slips and subsequent dissipation.
\end{abstract}

DOI: 10.1103/PhysRevE.66.026115

\section{INTRODUCTION}

When driven away from equilibrium, many systems encounter instabilities leading to additional states or phases. Often there exists a multiplicity of possible states that can be selected near the onset of the instability. The selected state may depend on various factors such as the rate at which the system is driven through the instability, noise, internal excitations, different dissipation mechanisms, and the system size.

In this paper the selection of states is studied in driven superconducting rings. Many of the phenomena observed here are not limited to superconducting rings, but appear in many other physical systems ranging from pattern forming systems [1-5] to lasers [6]. The relative simplicity of the superconducting system makes it possible to obtain information about some of the general questions in driven nonlinear systems such as state selection and the effect of dissipation on the state selection process itself.

The mesoscopic nature of the system, i.e., the superconducting ring having a finite circumference with a finite number of accessible states, is fundamental to this problem. First, it leads to the existence of a finite number of metastable current-carrying states which can compete for occupation. It is this competition that lies at the heart of the problem. Second, care must be taken to distinguish between voltagedriven and current-driven systems. As shown by Tarlie et al. [7], for systems that are not in the thermodynamic limit, i.e., mesoscopic systems, the choice of ensemble is not free. In this paper we focus on voltage-driven systems as opposed to current-driven systems.

In addition to providing a prototype system to study various aspects involving driven systems, in general, nonequilibrium superconductivity is of great interest in its own right. Indeed, the current-induced transitions in superconducting filaments have been a subject of intense experimental and theoretical study for almost three decades. Reference [8] provides a comprehensive review of the field.

We concentrate on the emergence of the dissipative phaseslip state [9-13] in voltage-driven mesoscopic systems.
PACS number(s): 02.50.-r, 74.40.+k, 05.45.-a, 74.60.Jg

When a superconductor (below $T_{c}$ ) is driven by a voltage source, the supercurrent increases until it reaches a critical value, at which point the system becomes unstable. Several interesting phenomena may then occur: the system will enter the dissipative phase-slip state, Joule heating can take place, mode locking, as well as other phenomena. Here, the focus is on the onset of the instability and its effect on the dynamics of the superconducting state.

The transitions between the current-carrying states can take place via two fundamentally different routes: (i) by a nucleation process involving thermal fluctuations and an activation energy barrier, or (ii) the system may be driven to an instability by an external driving force. In the context of nucleation and metastability, the decay of persistent currents in thin superconductors is an old and extensively studied problem [14-17]. However, the latter [13] involves a decay from a point of instability, and is relatively poorly understood. One of the major difficulties is this: whereas in the case of nucleation the decay is from a metastable state involving thermal activation and a saddle point, in the latter case the external force drives the system to a point of instability where there is no energy barrier left, i.e., the energy landscape looks locally flat. In this instance the decay and the final state depend on various factors, such as how fast the system was driven, the relative strength of fluctuations, internal excitations, and so on. This makes a precise theoretical formulation of the problem difficult, since it is not possible to use the free energy formulation as in the case of metastability [18].

\section{THE SYSTEM}

The physical system considered is a quasi-onedimensional superconducting ring of finite circumference, i.e., the radius of the cross-section area $S$ of the superconducting filament is much smaller than the coherence length $\xi$ and magnetic penetration length $\lambda: \sqrt{S} \ll \xi(T)$ and $\sqrt{S}$ $\ll \lambda(T)$, respectively, see Fig. 1(a). When the ring is placed in a time-dependent magnetic field, by Faraday's law of induction, an electromotive force (emf) is induced in the ring. 


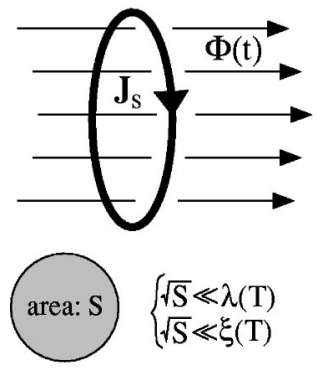

a)

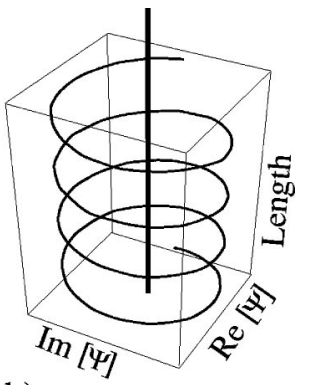

b)

FIG. 1. (a) Illustration of a voltage-driven superconducting ring. The magnetic flux is due to an infinitely long solenoid passing through the center of the loop. (b) Illustration of the currentcarrying states as uniformly twisted plane waves. At a phase slip, the amplitude of the helix approaches locally zero. The line through the center of the helix represents the superconducting wire.

From London's equation $\left(\vec{E}(\vec{r})=\partial_{t}\left[\left(4 \pi \lambda^{2} / c^{2}\right) \vec{J}_{s}(\vec{r}, t)\right]\right)$ this leads to a current that increases in time. (Here $\vec{E}$ is the electric field, $\vec{J}_{s}$ the supercurrent density, and $c$ the speed of light.) The time-dependent increase in the current cannot continue indefinitely, and eventually the current will reach a critical value, at which point the system becomes unstable and a dissipative phase slip will occur, resulting in a reduction of the current [13] by a discrete amount.

It is important to reemphasize that the system dynamics in the case under study here, viz., the decay of the system from a point of instability, is very different from the historically well-studied problem of the decay of the system from a point of metastability. The picture of the system hopping from one local minimum to the next no longer applies. Rather, the picture now is one where the system is initially in a locally stable state, but as a consequence of the voltage source, the energy landscape evolves in such a way that as the critical current is reached, the system finds itself at the top of a hill. When this situation is encountered, it is possible that there exist a variety of different valleys for the system to flow into, each valley leading to a locally stable state. In this picture, each of these locally stable states compete for occupation.

To examine this phenomena the Ginzburg-Landau (GL) theory of dirty superconductors will be considered. The Ginzburg-Landau free energy functional can be written as

$$
\begin{aligned}
\mathcal{F}[\Psi, \vec{A}]= & \int d \vec{x}\left\{\frac{\hbar^{2}}{2 m_{e}}\left|\left(\vec{\nabla}-i \frac{2 e}{\hbar c} \vec{A}\right) \Psi\right|^{2}+a|\Psi|^{2}+\frac{b}{2}|\Psi|^{4}\right\} \\
& +(8 \pi)^{-1} \int d \vec{x}(\vec{\nabla} \times \vec{A})^{2}
\end{aligned}
$$

where $\vec{A}$ is the vector potential, $\Psi$ is the complex valued order parameter, $e$ is the electron charge, $m_{e}$ is the electron mass, $c$ is the speed of light, $\hbar$ is Planck's constant, and $a$ and $b$ are the expansion coefficients. Since the current is induced in the loop by a time varying magnetic flux, the effect of the induced emf must be included in the GL description. By Faraday's law of induction, the electrons in the loop are subjected to an emf

$$
\mathcal{E}=-\frac{d \Phi(t)}{d t}=\oint \vec{E} \cdot d \vec{l}
$$

where $\mathcal{E}$ is the induced emf and $\Phi(t)$ is magnetic flux through the loop. The magnetic flux and the magnetic field are related by

$$
\Phi=\int_{\text {area }} \vec{B} \cdot d \vec{S}_{l}
$$

where $\vec{S}_{l}$ is the area of the loop. Equations (2) and (3) can be combined to obtain a relationship between the the vector potential and the electric field, i.e., if $E_{x}$ is used to denote the tangential component of the field, then $A_{x}=-E_{x} c t$, where $A_{x}$ is the tangential component of the vector potential. This in turn gives $A_{x}=-\mathcal{E}_{c} t / L$, where $L$ is the length of the wire.

The one-dimensional nature of the problem allows several simplifications. First, since the wire is narrow the magnetic field generated by the supercurrent does not significantly influence the order parameter. This allows one to treat the vector potential $A_{x}$ as a parameter instead of as a dynamical variable. In addition, since the magnetic field energy due to the supercurrent is much smaller than the energy associated with the order parameter, the magnetic field term can be dropped from the free energy [15]. Finally, since the radius of the wire is less than $\xi$ the order parameter is only a function of the tangential direction $(x)$. The geometry of the wire implies periodic boundary conditions, i.e., $\Psi(x)=\Psi(x+L)$.

For further analysis and computational efficiency it is convenient to rewrite the equation in a dimensionless form using the following transformations:

$$
\begin{gathered}
\Psi^{\prime} \equiv(b /|a|)^{1 / 2} \Psi, \\
x^{\prime} \equiv x / \xi, \\
A^{\prime} \equiv 2 e \xi A / \hbar c, \\
v_{e c}^{\prime} \equiv v_{e c} 2 e \tau_{G L} / \hbar,
\end{gathered}
$$

where $\xi^{2}=\hbar^{2} /\left(2 m_{e}|a|\right)$ and it is implicitly assumed that the temperature is below the superconducting transition (i.e., $a$ $<0) . \tau_{G L}$ is the Ginzburg-Landau time defined as

$$
\tau_{G L}=\frac{\pi \hbar}{8 k_{B}\left(T_{c}-T\right)},
$$

and it is the natural measure for time, i.e., $t \rightarrow t / \tau_{G L}$. In the following, we will work in dimensionless units, i.e., we perform the transformations as defined above and drop out the primes for convenience.

The last transformation in Eq. (4) involves $v_{e c}$, the electrochemical potential generated by the normal current, which will be formally introduced in the following section, where the GL theory is extended to include normal (Ohmic) current generation. In addition, following the scalings in Eqs. (4), it is natural to measure the length in units of the coherence length as $l=L / \xi$. Then the rescaled boundary condition reads $\Psi(x)=\Psi(x+l)$, and the dimensionless free energy becomes 


$$
\mathcal{F}=\int_{-l / 2}^{l / 2} d x\left\{\left|\left(\partial_{x}-i A_{x}\right) \Psi\right|^{2}-|\Psi|^{2}+\frac{1}{2}|\Psi|^{4}\right\}
$$

To describe the dynamics of the superconducting condensate, relaxational dynamics are assumed, leading to the standard stochastic time-dependent Ginzburg-Landau (STDGL) equation of motion, i.e.,

$$
\frac{\partial \Psi}{\partial t}=-\frac{\delta \mathcal{F}}{\delta \Psi^{*}}+\eta
$$

where $\eta \equiv \eta(x, t)$ is an uncorrelated Gaussian noise source with correlations

$$
\begin{gathered}
\langle\eta(x, t)\rangle=0, \\
\left\langle\eta^{*}(x, t) \eta\left(x^{\prime}, t^{\prime}\right)\right\rangle=2 D \delta\left(x-x^{\prime}\right) \delta\left(t-t^{\prime}\right) .
\end{gathered}
$$

The angular brackets denote an average, and $D$ is the intensity of the noise determined by the fluctuation-dissipation [17] theorem as

$$
D=\frac{2 \pi k_{B} T}{S H_{c}^{2} \xi}
$$

where $H_{c}$ is the critical field, and $H_{c}^{2} \propto(1-t)^{2}, \xi(T) \propto(1$ $-t)^{-1 / 2}$, and $t=T / T_{c}[28]$.

To make the model numerically more tractable, it is convenient to make the transformation $[17,19] \Psi \rightarrow \Psi e^{i q(t) x}$, where

$$
q(t)=A_{x}=\omega l^{-1} t,
$$

where $\omega=\tau_{G L} 2 e \mathcal{E} / \hbar$. This transformation twists, or winds, the order parameter along the wire. The effect of the transformation is to map the current-carrying states to twisted plane waves as illustrated in Fig. 1(b). After the transformation, the periodic boundary condition becomes

$$
\Psi(l+x, t)=\Psi(x, t) e^{i q(t) l},
$$

and the equation of motion obtained from Eq. (7) reads as

$$
\frac{\partial \Psi}{\partial t}=\frac{\partial^{2} \Psi}{\partial x^{2}}+\Psi-\Psi|\Psi|^{2}+i l^{-1} \omega x \Psi+\eta .
$$

This formulation neglects the electrochemical potential due to normal current generation at a phase-slip center. Its inclusion is discussed next.

\section{A. Electrochemical potential}

Equation (11) would be a sufficient description if the generation of a normal current at a phase slip could be neglected. This approximation is valid when the normal state resistivity is negligible $[13,17]$. However, the Ginzburg-Landau free energy is only valid for "dirty" superconductors in which the normal state resistivity is appreciable even at low temperatures. One aim of the current study is to examine the effect of the resistive normal current to the process. To facilitate this goal, the equation of motion [i.e., Eq. (11)] must be generalized to include the creation of electrochemical potential gradients at phase-slip locations.

A phase slip occurs when the system locally loses superconductivity and becomes a normal Ohmic conductor. As discussed above, below $T_{c}$ the system retains the fully superconducting state after making a transition to a state of lower current. An important question is the effect of the generation of normal current on the dynamics and the state selection problem.

To account for the generation of normal current, the time derivative in the STDGL equation of motion must be replaced by $\partial / \partial t+i v_{e c}$, where $v_{e c} \equiv v_{e c}(x, t)$ is the electrochemical potential generated by the normal current $[17,20-$ 23]. With that substitution, the dimensionless equation of motion becomes

$$
\left(\frac{\partial}{\partial t}+i v_{e c}\right) \Psi=\partial_{x}^{2} \Psi+\Psi-\Psi|\Psi|^{2}+i \frac{\omega x}{l} \Psi+\eta .
$$

Physically, the appearance of the electrochemical potential is due to local charge imbalance in a superconductor. Gorkov [24] was the first to point out that in a superconductor, the Fermi level, and thus the electrochemical potential, is a local time-dependent variable related to the coherence of the superconducting state. Qualitatively, if the local charge balance is disturbed, the Fermi level experiences a local time-dependent perturbation. This in turn affects the local energy gap. Gorkov showed that gauge invariance is preserved, if the order parameter depends on time as exp $\left(-2 i \mu_{F} t / \hbar\right)$, where $\mu_{F}$ is the Fermi energy. This leads to the second term on the left-hand side in Eq. (12).

The electrochemical potential can be determined by combining charge conservation and Ohm's law in the following manner. Charge conservation implies that $\partial_{x}\left(J_{n}+J_{s}\right)=0$, where $J_{n}$ is the normal current and $J_{s}$ is the supercurrent [25]. From Ohm's law, i.e., $\partial_{x} v_{e c}=-\alpha J_{n}$, this can be written as

$$
\frac{\partial^{2} v_{e c}}{\partial x^{2}}=\alpha \frac{\partial J_{s}}{\partial x}
$$

where $\alpha$ is a dimensionless Ohmic resistivity and can be written as

$$
\alpha=\rho_{n} / \rho_{o},
$$

where $\rho_{n}$ is the normal state resistivity,

$$
\rho_{o}=\frac{k_{B} T_{c}(1-t) \hbar}{\pi \xi(T)^{2} e^{2} H_{c}(T)^{2}}
$$

$t=T / T_{c}$, and $H_{c}(T)$ is the critical field. For a dirty superconductor [28] this can be written as

$$
\rho_{o}=0.1455 \frac{\hbar \mu_{o} k_{B} T_{c}}{\xi_{o} l_{F} e^{2} H_{c}^{2}(0)}
$$

where $l_{F}$ is the mean free path length. 


\section{LINEAR STABILITY ANALYSIS}

The aim of the linear stability analysis is to gain insight into the stability of the current-carrying state against small perturbations, how the perturbations grow or decay in time, and how different modes are selected. In general, when the total current exceeds the critical supercurrent, an Eckhaus instability occurs. The Eckhaus instability is a longitudinal secondary instability that appears in many systems exhibiting spatially periodic patterns [26,27].

To study the Eckhaus instability in superconducting rings the order parameter is linearized around a current-carrying state by setting $\Psi(x, t)=\Psi_{0}+\delta \Psi(x, t)$, where $\Psi_{0}$ $=\sqrt{1-q^{2}} e^{i q x}$ and $q=(\omega / l) t . \Psi_{0}$ is a current-carrying (or uniformly twisted plane wave) state that is a solution of Eq. (12) in the limit $\omega / l \ll\left(1-q_{c}^{2}\right)^{2} / q_{c} \approx 0.77$, where $q_{c}=1 / \sqrt{3}$. This limit is satisfied for the range of $\omega / l$ 's considered in this paper (i.e., $2 \times 10^{-6}<\omega / l<2 \times 10^{-3}$ ). Since the system possesses translational invariance and admits plane wave solutions, the perturbation is given in terms of its Fourier expansion, i.e.,

$$
\delta \Psi(x, t)=\sum_{n}\left[a_{k_{n}}(t) e^{i k_{n} x}+a_{-k_{n}}(t) e^{-i k_{n} x}\right] e^{i q x}
$$

where $a_{k_{n}}(t)$ is the amplitude of mode $n$ associated with wave vector $k_{n}=2 \pi n / l$. Substituting into Eq. (12), using Eqs. (9) and (13) to solve for $v_{e c}$, and linearizing in $\delta \Psi$ gives an equation of motion for $\delta \Psi$ or in Fourier space for $a_{k_{n}}$. Setting $a_{k_{n}}(t)=a_{k_{n}} e^{\lambda(q, \alpha) t}$ leads to an eigenvalue equation which can be solved to give

$$
\begin{aligned}
\lambda_{n}^{ \pm}(q, \alpha)= & -\left(1-q^{2}\right)(1+\alpha / 2)-k_{n}^{2} \pm\left[\left(1-q^{2}\right)^{2}(1-\alpha\right. \\
& \left.\left.+\alpha^{2} / 4\right)+4 q^{2}\left\{k_{n}^{2}+\alpha\left(1-q^{2}\right)\right\}\right]^{1 / 2} .
\end{aligned}
$$

When $\lambda_{n}^{ \pm}$is negative the corresponding mode is stable, fluctuations decay back to zero, and the superconducting state persists. When $\lambda_{n}^{ \pm}$is positive, the current-carrying states are unstable with respect to fluctuations of a finite wave vector $k_{n}$. For the following discussion, $\lambda_{n}^{-}$can be neglected as it is negative definite.

In Fig. $2, \lambda^{+}$is shown for the first three modes as a function of $q$ for several different values of $\alpha$. For small $q$ all the modes are stable, i.e., $\lambda_{n}^{+}<0$. The inset in Fig. 2(a) shows that the modes become unstable sequentially; the lowest mode first, then the mode $n=2$, and so on. The time $t_{n}$ at which a given mode becomes linearly unstable is determined by the condition $\lambda_{n}\left(t_{n}\right)=0$, which gives

$$
t_{n 1}=\frac{l}{\omega} \sqrt{1 / 3+k_{n}^{2} / 6} .
$$

For a wire of infinite length this time corresponds to the time at which the current reaches the critical value, i.e., $q_{n}$ $=(w / l) t_{n 1}=\sqrt{1 / 3+k_{n}^{2} / 6} \rightarrow \sqrt{1 / 3}$ and $J_{c}=q_{c}\left(1-q_{c}^{2}\right)=2 / \sqrt{27}$. While Eq. (18) implies that single phase-slip (i.e., $n=1$ ) processes will dominate, this effect is offset by the rate of increase of $\lambda_{n}^{+}$, i.e., $\partial \lambda_{n}^{+} / \partial q$ is an increasing function of $n$. This can be seen in the small- $\alpha$ limit, i.e.,

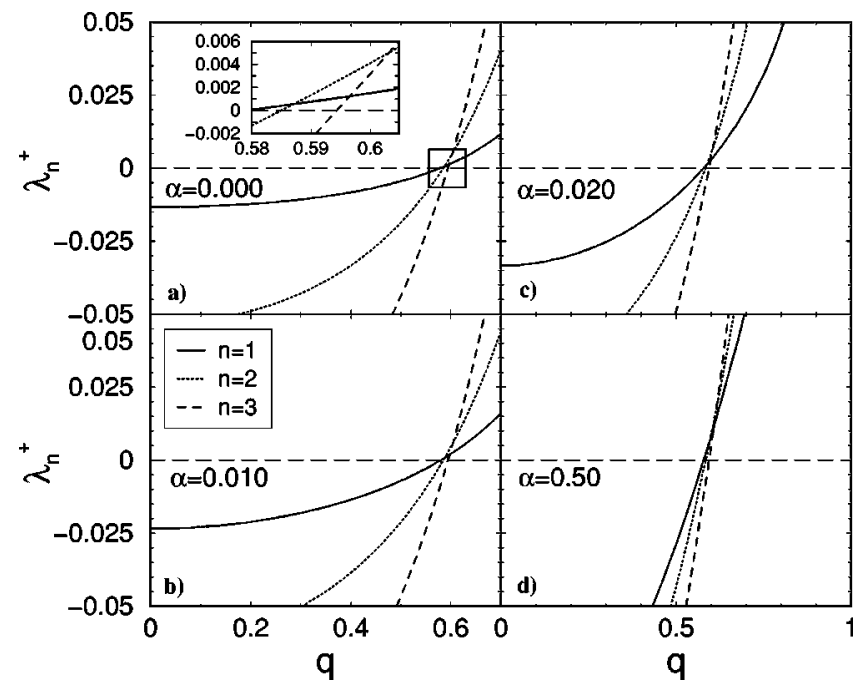

FIG. 2. The eigenvalues as a function $q(t)=\omega t / l$ for different $\alpha$ 's. The solid line is the $n=1$ mode, dotted line $n=2$ mode, and dashed line $n=3$ mode. For small $q$ all the modes are stable, i.e., $\lambda_{n}^{+}<0$, independent of the value of $\alpha$. Increasing the dissipation $\alpha$ increases the growth rate of all phase-slip modes. The inset in (a) shows the boxed area.

$$
\begin{aligned}
\left.\frac{\partial \lambda_{n}^{+}}{\partial q}\right|_{q_{n}}= & \frac{2 \sqrt{12+6 k_{n}^{2}}}{4+5 k_{n}^{2}}\left(3 k_{n}^{2}+\frac{\left(k_{n}^{2}+2\right)\left(4-k_{n}^{2}\right)}{4+5 k_{n}^{2}} \alpha\right. \\
& \left.+O\left(\alpha^{2}\right)+\cdots\right),
\end{aligned}
$$

or for simplicity in the small- $k_{n}$ limit,

$$
\left.\frac{\partial \lambda_{n}^{+}}{\partial q}\right|_{q_{n}}=\sqrt{3}\left(3 k_{n}^{2}+2 \alpha+\cdots\right) .
$$

Thus the rate of increase of the positive eigenvalue increases with $n$. The situation is somewhat analogous to the classic tortoise/hare race if only two modes are considered (say $n$ $=1$ and 2$)$. In this case the tortoise $(n=1)$ begins the race first since $t_{1}<t_{2}$, but the hare accelerates faster since $\partial \lambda_{1}^{+} /\left.\partial q\right|_{q_{1}}<\partial \lambda_{2}^{+} /\left.\partial q\right|_{q_{2}}$. To first order in $\alpha$, the effect of dissipation is to increase the rate of acceleration of both the tortoise and hare equally. Since the tortoise begins the race first this tends to favor the tortoise winning the race. In terms of mode analysis, increasing the dissipation (i.e., $\alpha$ ) increases the probability of a single phase slip $(n=1)$ occurring over a double phase slip $(n=2)$.

The linear predictions can be used to estimate the relative probabilities of a phase slip of order $n$ occurring. In the linear prediction the equal time correlation function for the $n$th mode is

$$
\left\langle\left|a_{n}(t)\right|^{2}\right\rangle=\frac{2 D}{l} e^{2 \sigma(t, \alpha)} \int_{0}^{t} d t^{\prime} e^{-2 \sigma\left(t^{\prime}, \alpha\right)},
$$

where 


$$
\sigma(t, \alpha) \equiv \int_{0}^{t} d t^{\prime} \lambda_{n}^{+}\left(t^{\prime}, \alpha\right)
$$

Following the instability, Eq. (21) describes the evolution of the $n$th mode from the initial current-carrying state described by $\Psi_{n}^{i}=\sqrt{1-q^{2}} \exp (i q x)$ to the new current-carrying state described by $\Psi_{n}=\bar{a}_{n} \exp \left[i\left(q-k_{n}\right) x\right]$, where $\bar{a}_{n}$ $=\sqrt{1-\left(q-k_{n}\right)^{2}}$. The quantity

$$
\hat{a}_{n} \equiv \sqrt{\left\langle\left|a_{n}(t)\right|^{2}\right\rangle} / \bar{a}_{n}
$$

describes the "distance" from the initial to the final $n$th state and can be thought of as an orthogonal coordinate in an $n$-dimensional space. The unit of measure in this space is then

$$
d=\sum_{n} \hat{a}_{n}^{2}
$$

If it is assumed that a phase slip has occurred when $d=1$, then it is natural to interpret the relative probability of an $n$th order phase slip as

$$
P_{n}=\frac{\hat{a}_{n}^{2}}{\sum_{n} \hat{a}_{n}^{2}} .
$$

Equation (25) provides a qualitative picture of the state selection process and makes it possible to compare the linear theory to numerical results. This will be done in Sec. IV (in particular, see Fig. 9).

In addition to the dependence of $P_{n}$ on $\lambda_{n}^{+}, \quad P_{n}$ also depends on the noise strength. While this is not directly visible from Eq. (25) it should be noted that the equation $d$ $=1$ imposes a $D$ dependence on $\hat{a}_{n}$ and $P_{n}$.

Physically, the noise strength depends on the temperature of the system via the fluctuation-dissipation theorem. The intensity of thermal noise increases as $T \rightarrow T_{c}$ as demonstrated by Eq. (8). Thus, close to $T_{c}$ the relative importance of the noise becomes increasingly important, whereas away for $T_{c}$ the driving force is dominant. Since $\alpha$ has no time dependence, the expansion of $\int_{0}^{t} d t_{1} \lambda_{n}^{+}\left(t_{1}, \alpha\right)$ leads to the same result as obtained by Tarlie and Elder [13], i.e., in terms of the intrinsic and extrinsic parameters, the instability of order $n$ becomes active at time $\tau_{n}=l\left(\partial_{q} \lambda_{n}^{+} \omega l\right)^{-1 / 2}$.

To summarize, the linear analysis shows that the state selection has a subtle dependence on both the applied driving force and on the intrinsic properties of the system. It is important to note that this analysis can only be expected to give a qualitative description of the process since the analysis does not account for competition between the various modes. These results will be compared with numerical simulations of the stochastic time-dependent GL equation in Sec. IV.

\section{NUMERICAL RESULTS}

The parameters that enter the numerical simulations can be estimated by considering typical experimental values,

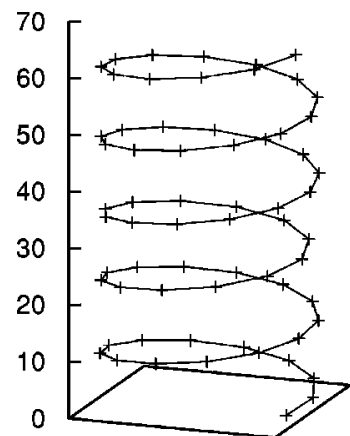

a)

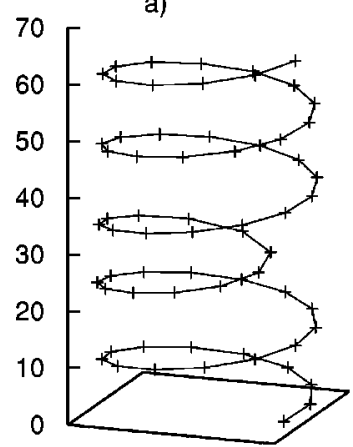

b)

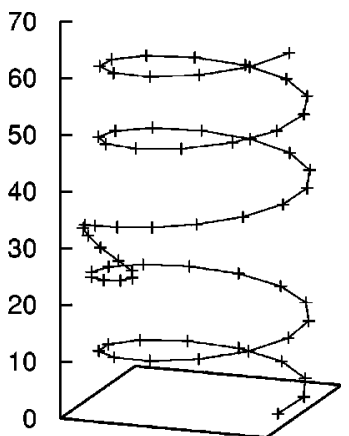

c)

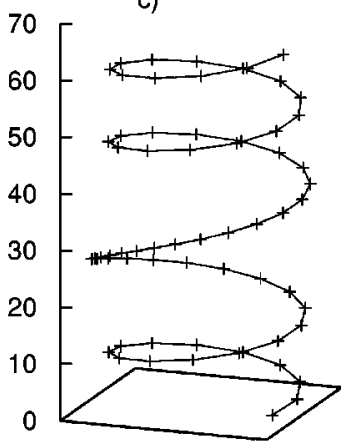

d)
FIG. 3. A snapshot of a phase-slip process with $L=64$ and $n_{l}$ $=5$. (a) The current-carrying states are uniformly twisted plane waves. (b) Due to fluctuations, the supercurrent at some location along the wire grows slightly faster than in the rest of the system. (c) At a phase slip the system makes a transition back to below the critical current by reducing the number of loops in the helix. (d) After the phase slip, the system retains the perfectly superconducting state with $J<J_{c}$ everywhere. $z$ axis, length, $x-y$ plane, $\operatorname{Re}[\Psi]$ and $\operatorname{Im}[\Psi]$.

such as $T_{c}=3 \mathrm{~K}, T=0.93 T_{c}, H_{c}=300 \mathrm{G}$, and $\xi(0)=\sqrt{S}$ $=1000 \AA$. With these values the intensity of the noise is $D=10^{-3}$, the GL time is $\tau_{G L}=1.4 \times 10^{-11}$ and $\omega$ $\approx \mathcal{E} / 23 \mu \mathrm{V}$. In the simulations the temperature is fixed and thus the intensity of noise is fixed. $\omega$ was varied between 0.0001 and 0.1 . This corresponds to electromotive forces from $2 \mathrm{nV}$ to $2 \mu \mathrm{V}$. For dirty superconductors the normal state resistivity can vary between 0.01 and $1.0 \mu \Omega \mathrm{cm}$ and $\rho_{o}$ varies from 1.0 to $100.0 \mu \Omega \mathrm{cm}$. Using these values the dimensionless resistivity, $\alpha \approx 10^{-4}-1.0$, depending on the dimensions and the material.

A simple Euler algorithm was used for the time integration of Eq. (12), and Eq. (13) was solved in Fourier space. The complex order parameter was separated into its real and imaginary parts. The simulation parameters were $L$ $=64, d x=0.85, d t=0.2$, where $d x$ and $d t$ are the smallest discrete elements of space and time, respectively.

A useful parametrization of the length of the system is $n_{l} \equiv l q_{c} / 2 \pi$, where $q_{c}=1 / \sqrt{3}$ from the Eckhaus analysis of the GL equation as discussed above. $n_{l}$ is interpreted as the winding number of the order parameter when the Eckhaus instability is encountered. For the simulations to follow, $n_{l}$ $=5$ (see Fig. 3). This allows enough complexity due to interaction between different modes, i.e., five modes can compete for occupation, while remaining numerically tractable. 


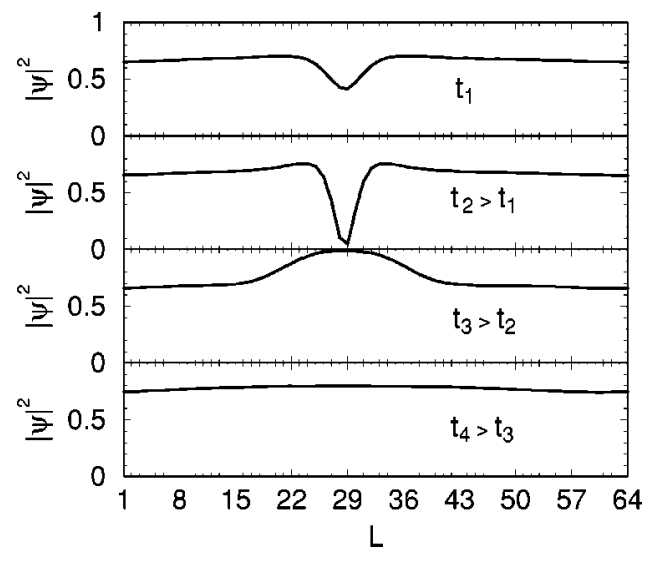

FIG. 4. Order parameter just before and after the phase slip. As $|\Psi| \rightarrow 0$, the phase gradient must grow in order to maintain a constant current. As the amplitude goes to zero, it can "slip" by a multiple of $2 \pi$ and relax to a state of lower current.

When computing the probability of an $n$ th-order phase slip, $P_{n}$, the averaging was typically from 2000 phase-slip events (small $\omega$ ) up to 15000 phase slips (large $\omega$ ). Simulations performed at large values of $\alpha$ and $\omega$ (not shown here) often lead to unusual results, which may be due to numerical inaccuracies.

\section{A. Dynamics of the order parameter}

The dynamics of the order parameter around a phase slip is illustrated in Figs. 3 and 4. Figure 3 illustrates that a current-carrying state is a uniformly twisted plane wave. As the current increases, the helix becomes more tightly wound. Due to fluctuations, there will be weak spots where the local supercurrent reaches the critical current before the rest of the system. This is the point where the amplitude of the order parameter starts to decay rapidly toward zero. When $|\Psi|^{2}$ $\rightarrow 0$, the phase-slip center momentarily disconnects the phases to the left and right of it, the helix looses a loop, and the supercurrent jumps to a lower value. This cycle is repeated periodically.

In Figs. 3 and 4 the behavior described using linear analysis in the preceding section is clearly visible: as the supercurrent increases, the absolute value of the order parameter, $|\Psi|^{2}$, decreases and, at the moment of the phase slip, approaches zero. After the phase slip the order parameter rapidly recovers. Figure 4 demonstrates this behavior. This allows the amplitude to relax toward equilibrium in the vicinity of the phase-slip center [times $t_{2}$ and $t_{3}$ in Figs. 4 and 3(c) and 3(d), respectively]. After a short time the wire obtains a uniform current ( $t_{4}$ in Fig. 4$)$.

To quantify the phase-slip events it is useful to consider two quantities: the spatially averaged supercurrent and the winding number, both at time $t$. The spatially averaged supercurrent is given by

$$
J_{s}(t)=\frac{1}{2 l} \int_{0}^{l} J_{s}(\Psi) d x .
$$

The winding number is a measure of the total phase change in the system and can be defined as

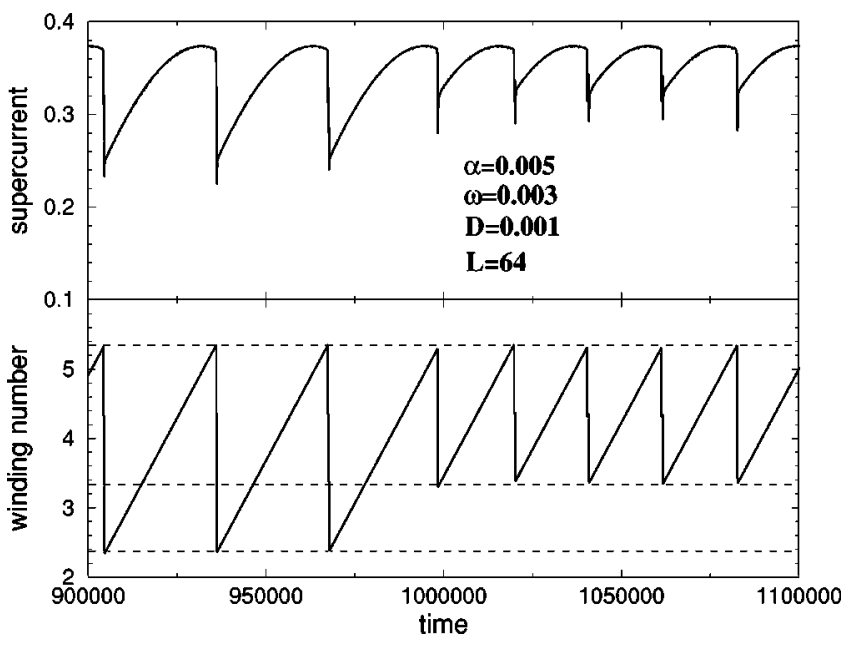

FIG. 5. Supercurrent and winding number as a function of time. When this figure is compared to the state selection probabilities in Fig. 6, it can be seen that the probabilities of double and triple phase slips are almost equal, but there is still a small probability for single slips.

$$
W(t)=\frac{1}{2 \pi} \int_{0}^{l} d x \frac{\partial[\arg (\Psi(x, t))]}{\partial x} .
$$

As described above, the order parameter can change its winding number by $2 \pi n$, where $n= \pm 1, \pm 2, \ldots$ Only changes by an integral multiple of $2 \pi$ are possible in order to preserve the continuity of the order parameter. This also implies that at a single (multiple) phase slip, the system removes exactly one (integral multiple) fluxoid.

Figure 5 displays the time development of the supercurrent and winding number defined in Eqs. (26) and (27), respectively. The electric field drives the current to the critical current, where an instability occurs and the current jumps to a lower value. As suggested by Fig. 5, there can be several modes simultaneously present. In the figure, phase slips of order two dominate but occasionally there are jumps of order three. The relative occurrence of phase slips of all orders is shown in Fig. 6 as a function of driving force (i.e., $\omega$ ) for several values of $\alpha$.

As discussed in connection with the linear stability analysis the appearance of phase slips of different orders is a subtle issue. For example, every now and then the winding number displays little dips, as if the total phase slip was a result of a two-stage process. It is instructive to look at the state selection probabilities in Fig. 6 together with the dynamics of the supercurrent and the winding number in Fig. 5. As seen from Fig. 6 phase slips of order $n=1$ dominate the process at low driving forces. As the driving force is increased phase slips of order $n=2$ become dominant and the shape of the probability curve becomes skewed. Order by order, other modes become dominant in a similar manner. This is consistent with the linear stability analysis as shown in Fig. 2.

The little dips referred to above are a result of competition between the modes. As seen in the linear stability analysis, modes of lower order become unstable first but the higher- 


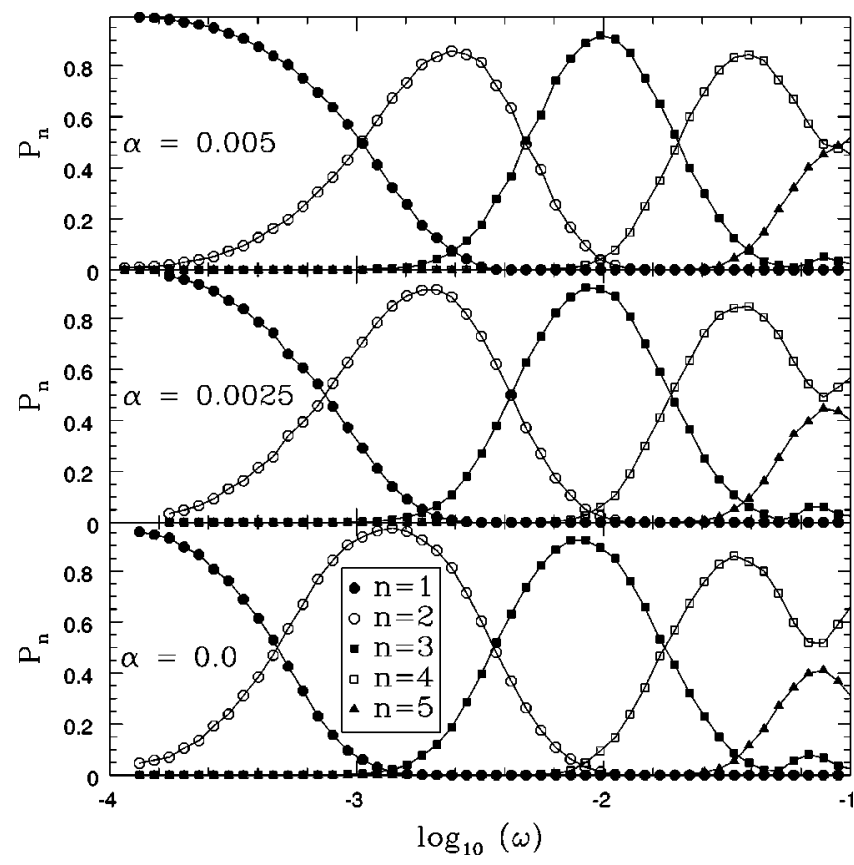

FIG. 6. State selection probabilities from numerical simulations as a function of the driving force. The closed circles denote single phase slips, open circles, double; closed squares, triple; open squares, quadruple; and closed triangles, phase slips of order five.

order ones grow at a faster rate. This leads to competition and crossover effects. This implies that the dips in Fig. 5 are not due to a result of a two-stage process, where a phase slip of higher order occurs via two lower-order processes, but instead due to the coexistence of different modes with different growth rates. Figure 7 illustrates the complicated nature of the phase slip when several modes are simultaneously present. There is a competition between different modes, and it is even possible for several phase-slip centers to exist (almost) simultaneously.

Figure 8 shows the time rate of change of $|\Psi|^{2}$ and the electrochemical potential at the phase-slip center as a function of time, when the mode $n=1$ is dominant. The time frame is selected in such a way that the figures cover the immediate vicinity of the phase slip. At the moment of the phase slip, $|\Psi|^{2}=0$. After the phase slip, $\Psi$ rapidly recovers its equilibrium value. Since there is a constant emf acting on the superconductor, $|\Psi|$ starts to decrease after its recovery. As seen in the lower figure, $v_{e c}$ regains its equilibrium value $\left(v_{e c}=0\right)$ at the phase-slip center considerably slower than $\Psi$. This can be seen in the following way. The electrochemical potential is zero if the current is uniform throughout the sample. However, as seen in Figs. 4 and 7, the time required to reach a uniform current is much longer than the time required for healing of the order parameter at the phase-slip center. Physically, this corresponds to relaxation of the charge imbalance [28] in a superconductor. The relaxation is diffusive $[8,10,29]$, with time scales typically of order $10^{-9}$ $-10^{-10} \mathrm{~s}$.

As discussed in the preceding section the electrochemical potential, or dissipation, changes the probability of making an $n$th order phase slip. This can be seen in Fig. 6 for all
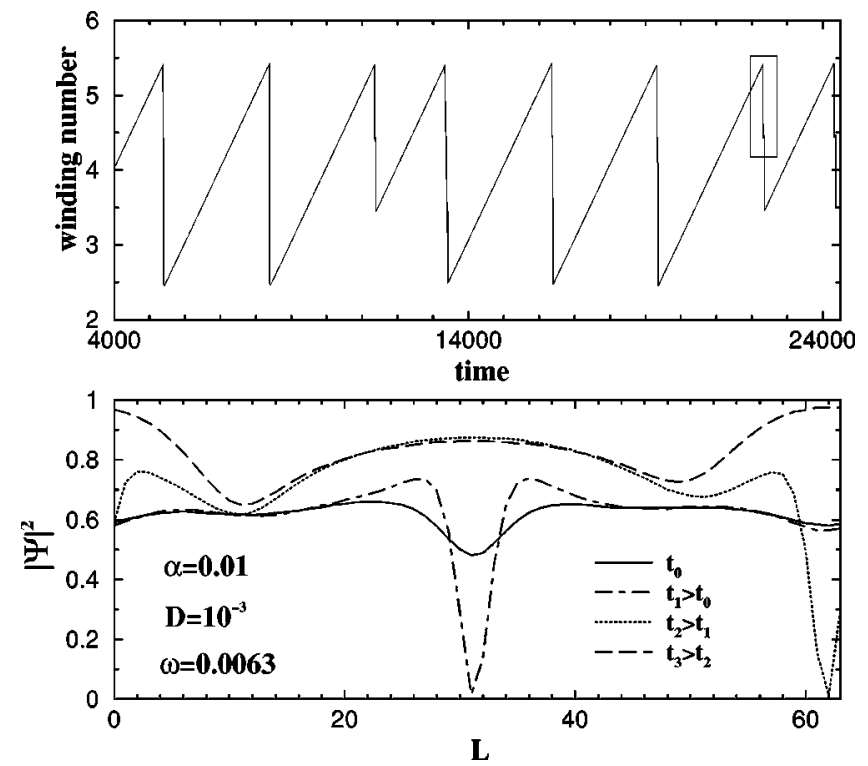

FIG. 7. Top: The winding number as a function of time. Bottom: The square of the amplitude of the order parameter taken at four different times inside the boxed area. As the winding numbers increases, there are spots where the amplitude starts to decay $\left(t_{0}\right)$. This leads to competition and coexistence of several modes, and several possible phase-slip centers. The parameters correspond to the case when three modes, $n=1,2,3$, are present.

values of $\omega$. To highlight this feature the selection probabilities were numerically estimated as a function of $\alpha$ for $\omega$ $=10^{-3}$ and are displayed in Fig. 9. In this figure the linear prediction [i.e., Eqs. (21)-(25)] is also included for comparison. While the linear analysis fails to predict the correct amplitudes for different modes, it provides the correct qualitative picture and predicts the order in which different modes become dominant. The quantitative discrepancies stem from

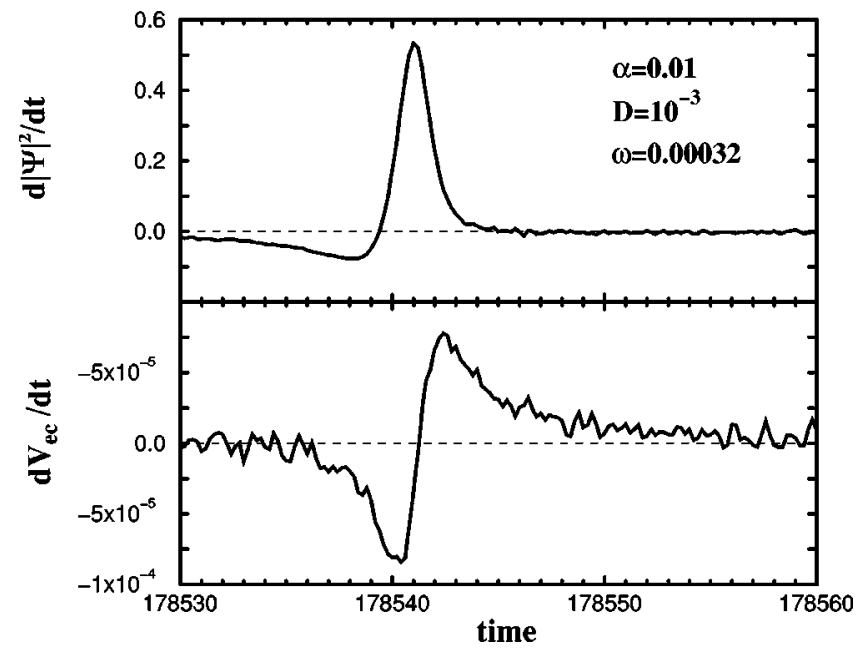

FIG. 8. Top: The time derivative of $|\Psi|^{2}$ at the location of the phase slip as a function of time. Bottom: The rate of change of the electrochemical potential at the phase-slip center as a function of time. The simulation parameters correspond to a case when single slips dominate almost completely. The slice runs from immediately before the phase slip to just after it. 


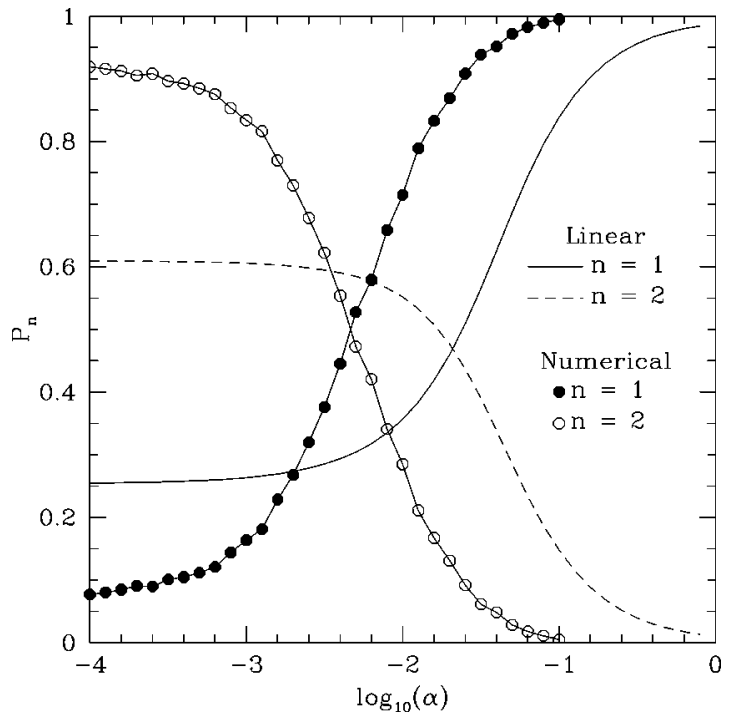

FIG. 9. State selection probabilities as a function of the dissipation (i.e., $\alpha$ ) for $\omega=10^{-3}$.

two factors, first the nonlinear terms seem to favor a separation between the modes. Figure 6 shows that once a mode becomes dominant, it quickly suppresses all the other ones. However, in linear theory all the allowable modes have much higher amplitudes at all values of the driving force $\omega$ [30]. Second, at the limit $\alpha \rightarrow 0$ the linear theory predicts accurately the crossover points where a new mode becomes dominant [30], but the presence of dissipation (finite $\alpha$ ) has a significant effect on it as can be seen in Figs. 6 and 9.

\section{B. Power dissipated at a phase slip}

A phase slip is a dissipative process where electrical energy is locally converted into heat. This is due to Ohmic resistance at the phase-slip center. Early experiments [10,29] showed that the differential resistance related to the phase slip is temperature independent for a wide range of temperatures except very close to $T_{c}$. This is a delicate issue; heating due to Ohmic resistance changes the local critical current, and issues related to charge imbalance and relaxation may become important [8]. In the following, the heat generated at a phase slip is estimated. The normal carriers are assumed to follow Ohm's law.

The Joule heating law can be used to estimate the heat generated at a phase slip. The power generated is

$$
P=\int_{\text {volume }} \vec{j}_{n} \cdot \vec{E} d \Omega=S \int j_{n} E_{x} d x,
$$

where $d \Omega$ is a volume element, $S$ is the cross-sectional area of the ring, $\vec{j}_{n}$ is the dimensional normal current density, and $E_{x}$ is the electric field along the wire. In terms of the electrochemical potential, the energy per unit volume can then be written as

$$
\frac{E}{V}=\mathcal{E}_{o} \int_{0}^{\tau}\left[\int_{0}^{l}\left|\vec{J}_{n}\right|^{2} d x\right] d \tau^{\prime},
$$

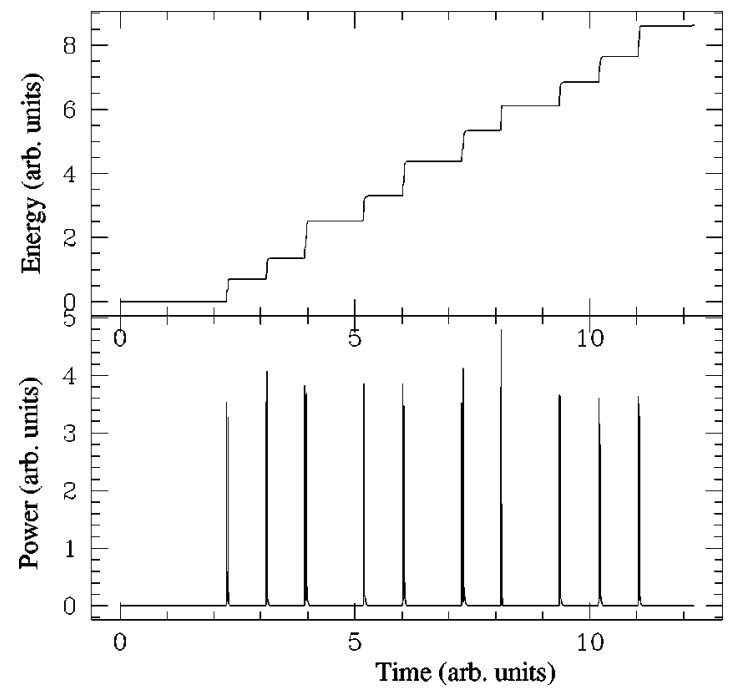

FIG. 10. Top: Energy (in arbitrary units) dissipated as a function of time. Bottom: The corresponding power (in arbitrary units) dissipated at phase slips. The figures show clearly the quantized nature of dissipation. The crossover effects are visible in the width of peaks. The simulation parameters were $d x=0.85, d t=0.2, \alpha$ $=0.01, \omega=0.0064$, and $D=10^{-3}$. These parameters correspond to a case when three modes $(n=1,2,3)$ are active at the same time.

where $\mathcal{E}_{o}=\left(2 H_{c}^{2} / l\right) \alpha$ and $\vec{J}_{n}$ is the dimensionless normal current density.

The increase in temperature due to a phase slip can be estimated using the heat capacity. The heat capacity per unit mass is

$$
c=\frac{1}{m} \frac{\Delta E}{\Delta T}
$$

where $c$ is the specific heat. The change in temperature is then

$$
\Delta T=T_{0} \int_{0}^{\tau}\left[\int_{0}^{l} J_{n}^{2} d x\right] d \tau^{\prime}
$$

where

$$
T_{0}=\frac{2 H_{c}^{2}}{l c \rho_{m}} \alpha,
$$

$\rho_{m}$ is the mass density, and $H_{c}^{2} \propto(1-t)^{2}$, where $t=T / T_{c}$ [28]. Equation (30) can be used to estimate the change in temperature due to a phase slip. The linear dependence of $T_{0}$ on $(1-t)^{2}$ expresses the well-known fact $[10,29]$ that close to $T_{c}$ the effects of Joule heating are less significant. Evaluation of $\Delta T$ requires information about the time and the length scales involving $v_{e c}$, and therefore we have not estimated it here. Figure 10 shows the accumulated energy and power dissipated as a function of time.

\section{CONCLUSION}

Here, the dynamics of accelerated quasi-one-dimensional superconductors under the influence of a voltage source was 
studied. A constant emf was used to accelerate the supercurrent to the critical current, at which point the Eckhaus instability is encountered and multiple metastable states can compete for occupation. Each of these competing metastable states corresponds to a state with a different supercurrent. The transition to a new state of lower current involves generation of a resistive phase-slip center that heals after the phase slip. Because the system was driven by a voltage source, it allowed the study of a very general phenomenon, namely, the relation to the general methods and problems in nonlinear dynamics, statistical mechanics, and pattern formation.

Linear stability analysis was used to investigate the Eckhaus instability. It was found out that within the linear approximation, the state selection process is a competition of two factors: the characteristic time at which a mode $a_{n}(t)$ becomes unstable, and the growth rates of the other modes. For small driving forces, the low-order modes have time to grow and dominate the process, whereas for larger driving forces the faster growth rates of high-order modes lead to their dominance. In the intermediate region the competition leads to crossover.

Numerical simulations were performed by simulating the stochastic time-dependent Ginzburg-Landau equation. It was found out that the behavior is consistent with the predictions of the linear analysis. Although the behavior was qualita- tively similar, nonlinearities and interaction between the phase slips at higher driving forces and higher normal current resistivity lead to differences.

In spite of the simplicity of the system, it displays rich and complex phenomena, and more analytical and numerical studies are needed. To the authors' knowledge, there exists no systematic method to study state selection in accelerated systems. Recent work $[18,30]$, suggests that the path integral method of Onsager and Machlup [31] may offer a framework for a systematic study of the decay of systems from points of instability when multiple modes compete for occupation. The extension of this work to problems where the dynamical system is evolving in time, as is the case here, has not been explored. Additionally, future work could explore the twodimensional case numerically.

\section{ACKNOWLEDGMENTS}

This work has been supported by the Academy of Finland (M.K.), the Finnish Cultural Foundation (M.K.), the Finnish Academy of Science and Letters (M.K.), Research Corporation Grant No. CC4787 (KRE), NSF-DMR Grant No. 076054 (KRE), the Natural Sciences and Engineering Council of Canada (M.G.), and le Fonds pour la Formation de Chercheurs et l'Aide à la Recherche du Québec (M.G.).
[1] L. Kramer and W. Zimmermann, Physica D 16, 221 (1985).

[2] E. Ben-Jacob, H. Brand, G. Dee, L. Kramer, and J. S. Langer, Physica D 14, 348 (1985).

[3] L. Kramer, H. R. Schober, and W. Zimmermann, Physica D 31, 212 (1988).

[4] B. Caroli, C. Caroli, and B. Roulet, in Solids Far from Equilibrium, edited by C. Godrèche, (Cambridge University Press, Cambridge, 1992), p. 155.

[5] E. Hernández-García, J. Vinãls, R. Toral, and M. San Miguel, Phys. Rev. Lett. 70, 3576 (1993).

[6] M. C. Torrent and M. San Miguel, Phys. Rev. A 38, 245 (1988).

[7] Martin B. Tarlie, E. Shimshoni, and P. M. Goldbart, Phys. Rev. B 49, 494 (1994).

[8] R. Tidecks, Current Induced Non-equilibrium Phenomena in Quasi-One-Dimensional Superconductors, Springer Tracts in Modern Physics, Vol. 121 (Springer-Verlag, Berlin, 1990).

[9] T. Rieger, D. J. Scalapino, and J. E. Mercereau, Phys. Rev. B 6, 1734 (1972).

[10] W. J. Skocpol, M. R. Beasley, and M. Tinkham, J. Low Temp. Phys. 16, 145 (1974).

[11] L. Kramer and A. Baratoff, Phys. Rev. Lett. 38, 518 (1977).

[12] L. Kramer and R. Rangel, J. Low Temp. Phys. 57, 391 (1984).

[13] M. B. Tarlie and K. R. Elder, Phys. Rev. Lett. 81, 18 (1998).

[14] W. A. Little, Phys. Rev. 156, 396 (1967).

[15] J. S. Langer and V. Ambegaokar, Phys. Rev. 164, 498 (1967).
[16] D. E. McCumber, Phys. Rev. 172, 427 (1968).

[17] D. E. McCumber and B. I. Halperin, Phys. Rev. B 1, 1054 (1970).

[18] Alan McKane and Martin Tarlie, Phys. Rev. E 64, 026116 (2001).

[19] N. Byers and C. N. Yang, Phys. Rev. Lett. 7, 46 (1961).

[20] M. J. Stephen and H. Suhl, Phys. Rev. Lett. 13, 797 (1964).

[21] P. W. Anderson, N. R. Werthamer, and J. M. Luttinger, Phys. Rev. 138, A1157 (1965).

[22] A. Schmid, Phys. Kondens. Mater. 5, 302 (1966).

[23] E. Abrahams and T. Tsuneto, Phys. Rev. 152, 416 (1966).

[24] L. P. Gorkov, Zh. Eksp. Teor. Fiz. 34, 735 (1958) [Sov. Phys. JETP 7, 505 (1958)].

[25] For the one-dimensional wire considered in this paper the dimensionless supercurrent in given by $J_{s}=\left(\psi^{*} \partial \psi / \partial x\right.$ $\left.-\psi \partial \psi^{*} / \partial x\right) / 2 i$.

[26] W. Eckhaus, Studies in Non-Linear Stability Theory, Springer Tracts in Natural Philosophy, Vol. 6 (Springer-Verlag, New York, 1965).

[27] M. A. Dominguez-Lerma, D. S. Cannell, and G. Ahlers, Phys. Rev. A 34, 4956 (1986).

[28] M. Tinkham, Introduction to Superconductivity, 2nd ed., International Series in Pure and Applied Physics (McGraw-Hill, New York, 1996).

[29] G. J. Dolan and L. D. Jackel, Phys. Rev. Lett. 39, 1628 (1977).

[30] M. B. Tarlie and A. J. McKane, J. Phys. A 31, L71 (1998).

[31] L. Onsager and S. Machlup, Phys. Rev. 91, 1505 (1953). 